\title{
Zur Förderung der akademischen Textkompetenz in der Ausbildung der DaF-Lehrkräfte ${ }^{1}$
}

\author{
Nurcihan Sönmez Genç (D), Ankara - D. Çiğdem Ünal (iD, Ankara
}

https://dx.doi.org/10.37583/diyalog.958505

\begin{abstract}
Deutsch)
Das Ziel dieses Artikels ist es aufzuzeigen, wie die akademische Textkompetenz von angehenden DaFLehrkräften in der Türkei im Rahmen des Grundstudiums gefördert werden kann. Anknüpfend an das Unterrichtsprinzip der Prozessorientierung und den Prozeduren des 3-Phasen-Modells zur Förderung der Textkompetenz von Schmölzer-Eibinger (2007) werden unterschiedliche unterrichtspraktische Beispiele präsentiert, die von den Forscherinnen dieser Arbeit, mit dem Ziel zur integrativen und prozessorientierten Förderung der akademischen Textkompetenz von angehenden DaF-Lehrkräften im Rahmen des Wahlfaches „Akademisches Deutsch“ des aktuellen Curriculums der Deutschlehrerausbildung entwickelt wurden. Mit dem Einsatz der präsentierten Unterrichtsbeispiele wird ein Mechanismus der Textarbeit in Gang gesetzt, durch die jene kognitiven Prozesse angeregt und intensiviert werden, die im Umgang mit Texten besonders produktivitätssteigernd und lernwirksam sind. Um die Studierenden der Deutschlehrerausbildung gezielt auf die fachlichen Inhalte des Grundstudiums vorbereiten zu können, gilt es, ihnen das ,Know-How ‘ der Textproduktion durch das gezielte Einbringen von didaktisch begründeten Unterrichtseinheiten in die universitäre Unterrichtspraxis verfügbar zu machen.
\end{abstract}

Schlüsselwörter: Förderung der akademischen Textkompetenz, Prozessorientierung, Deutschlehrerausbildung, Akademisches Deutsch als Wahlfach, Deutsch als Fremdsprache.

\section{Abstract (English) \\ Promotion of academic writing competence in German Teacher Education Programs}

The purpose of this study is to demonstrate how the academic German text competence of pre-service German teachers being trained in universities can be developed with a process-oriented learning environment where the content of the teaching program, they are subject to, is integrated. Within this context, in this study, a detailed explanation will be provided as to how the practices with regard to the development of this key skill which is of great importance for learning success can be integrated and applied in the "Academic German" elective course in current German Teaching Program. This study presents a variety of samples that are designed for the purpose of developing the academic text competence of pre-service German teachers based on the principle of process-orientation and the in-class procedures of the three stage model that was developed by Schmölzer-Eibinger (2007) for the improvement of text competence. With the use of the presented teaching examples, a text work

Einsendedatum: 27.03.2021

Freigabe zur Veröffentlichung: 30.06.2021

${ }^{1}$ Die vorliegende Artikel basiert auf der Dissertation der Erstautorin mit dem Titel „Eine Studie zur Förderung der akademischen Textkompetenz im Kontext der DaF-Lehrerausbildung“, die von der Zweitautorin betreut wurde. 
mechanism is set in motion that stimulates and intensifies those cognitive processes that are particularly productivity-enhancing and effective in learning when dealing with texts. In order to be able to prepare the students of the German language teaching for the content knowledge of the teacher training program, it is necessary to make the know-how of the text production available to them through the undergraduate courses.

Keywords: development of academic text competence, process-orientation, German Teacher Education, "Academic German" as en elective Course, German as a foreign language. 


\section{EXTENDED ABSTRACT}

The term academic text competence is understood to mean the abilities and skills that an individual needs in order to use texts for knowledge acquisition and knowledge processing and to be able to successfully write their own texts. In order to be able to acquire the professional skills required for the teaching profession, pre-service teachers/teacher candidates are required to develop their academic text skills adequately in accordance with the written requirements of the respective teacher training course.

This study first addresses the questions of what is understood by the term academic text competence and in which linguistic register this competence can be located. The importance of this key competence for educational success in the context of a GFL- teacher education program is also to be highlighted. Then the integration of the elective university course "Academic German" into the current German teacher training curriculum will be explained in more detail. This will be followed by a presentation of the way that process-oriented work can be carried out to promote academic text competence in the "Academic German" course in question and the methodological and didactic approaches that can be preferably used in this regard. In addition, following the teaching principle of process orientation and the procedures of the 3-phase model developed by Schmölzer-Eibinger (2007) for the promotion of text competence, a variety of practical teaching examples will be presented. Those teaching samples are designed by the researchers of this thesis for the purpose of promoting the academic text competence of prospective GFL- Teachers in Turkey, and then are applied, tested, and optimized in the elective course "Academic German" in German teacher education program. The main aim of the 3-phase model is to make a variety of necessary tools accessible to the learners in all the sub-processes of text production, which help them successfully master the individual text production phases. It is shown that while writing a text, orientation towards the production logic of a text and the stepby-step completion of the individual text production phases is extremely useful and increases productivity. Schmölzer-Eibinger characterizes (2008: 33) the 3-phase model for promoting text competence as a learner-oriented, versatile, easy-to-use didactic model that can be used in a wide variety of learning contexts. The model comprises three systematically related and networked components. The first phase of knowledge activation aims to call up the thoughts, associations, spontaneous ideas and the existing experiences and knowledge of the learners on a specific topic and to make them available for work on a text (Schmölzer-Eibinger 2008: 29ff). The second phase, working on the texts, is the key area of the 3-phase model. Essentially, the task arrangements in this phase aim to encourage the learners to perceive texts from different perspectives and to reflect on, reconstitute, revise or reconstitute them in different contexts. The work on the texts takes place in three stages: text construction, text reconstruction, text focus and text expansion. The third phase of the 3-phase model for promoting text competence is about removing texts from their original contexts and transferring them to new contexts. It should be emphasized that on closer inspection of the 3-phase model, it quickly becomes clear that the process character of text production serves as a sequential system for teaching and that the principle of process orientation is 
guaranteed through the targeted interlocking of the three phases. The targeted activation of knowledge in the first phase creates an initial basis for the subsequent text work phase. The reconstructive-productive text processing advanced in small steps in the second phase in turn provides an impetus for the new conception of texts in the third phase. Through the interaction of the individual functions of this model, a text work mechanism is set in motion through which those cognitive processes that are particularly productivity-increasing and effective in learning when dealing with texts are stimulated and intensified (Schmölzer-Eibinger 2008: 33).

Based on the requirement for the development of specific methodological and didactic aids, which are tailored to the problem at hand, practical and directly applicable, the aim of the present work with the presentation of the teaching examples presented is to make a concrete contribution to promoting the academic text competence of prospective GFL- teachers. 


\section{Einleitung}

Die Fähigkeit eines Individuums, die eigenen an der Schriftsprache orientierten sprachlichen Kompetenzen an die sich stetig verändernden Heraus- und Anforderungen des gegenwärtigen Arbeitsmarktes anzupassen, bildet ein konstitutives Element der beruflichen Handlungskompetenz (vgl. Ohm 2014: 7). Angesichts der Tatsache, dass ohne eine sprachliche Qualifizierung auch keine berufliche Qualifizierung erreicht werden kann, kristallisiert sich die zunehmende Bedeutung einer zeitgemäßen und qualitativ hochwertigen Fremdsprachenlehrerausbildung heraus. Folglich gilt es, die Effizienz und Effektivität dieser Studiengänge unter Beachtung der gegenwärtigen und zukünftigen Entwicklungen stetig zu steigern (vgl. Bausch 1992: 24; Krumm 2014: 8; Seferoğlu 2004: 40; Tapan 2000: 38).

Der Fokus der vorliegenden Arbeit liegt in diesem Zusammenhang auf der Betrachtung der deutschsprachigen DaF-Lehramtsstudiengänge in der Türkei. So ist zu betonen, dass eine qualifizierte DaF-Lehrkraft neben einer ausreichend entwickelten Sprachkompetenz im Deutschen auch über eine Fachkompetenz verfügen muss, welche auf einem ausreichend entwickelten Fachwissen, fachdidaktischen Wissen und einem pädagogischen Wissen gründet (vgl. Lipowsky 2006: 49). Demgemäß muss eine DaFLehrkraft am Ende ihres Studiums neben guten Deutschkenntnissen auch über ein umfassendes theoretisches Fachwissen verfügen, so dass er oder sie sich mit den grundlegenden Fragestellungen im Fachbereich der Methodik und Didaktik des Deutschunterrichts auseinandersetzen kann. Der Zugang zu diesem theoretischen Fachwissen gründet im tertiären Bildungsbereich hauptsächlich auf der kompetenten Nutzung von verschriftlichten Wissensbeständen (Cummins 1999). Übertragen auf die deutschsprachige DaF-Lehrerausbildung in der Türkei bedeutet dies, dass die Vermittlung der fachspezifischen Studieninhalte grundsätzlich auf der Basis von unterschiedlichen Fachtexten erfolgt. Bei diesen Fachtexten kann es sich beispielsweise um theoriebezogene Textsorten, wissenschaftspraktisch - didaktische Textsorten oder auch um studentische Schrifttexte handeln (siehe Heinemann 2000: 705). Im Rahmen eines DaF-Lehramtsstudiums bildet folglich das Verfügen über eine adäquat ausgebildete akademische Textkompetenz die Grundvoraussetzung für einen souveränen Umgang mit deutschsprachigen Fachtexten (vgl. Decker u.a. 2015: 2; Franken 2020: 2).

\section{Ziel und Fragestellung}

Das Ziel dieses Artikels ist es, die akademische Textkompetenz und deren Bedeutung für den Bildungserfolg im Kontext eines deutschsprachigen DaF-Lehramtsstudiums zu beleuchten. Vor diesem Hintergrund wird die Integration des neuen Seminars Akademisches Deutsch in das aktuelle Curriculum der DaF- Lehrerausbildung näher erläutert und es werden anknüpfend an das Unterrichtsprinzip der Prozessorientierung und den Prozeduren des 3-Phasen-Modells zur Förderung der Textkompetenz von Schmölzer-Eibinger (2007) unterrichtspraktische Beispiele präsentiert, die von den Forscherinnen dieser Arbeit, mit dem Ziel zur Förderung der akademischen 
Textkompetenz von angehenden DaF-Lehrkräften in der Türkei, entwickelt und erprobt wurden. Mit der Darlegung unterschiedlicher didaktisch begründeter Beispiele konzentriert sich dieser Artikel auf die Beantwortung folgender Fragen:

- Welcher methodisch-didaktische Ansatz bietet sich für die Förderung der akademischen Textkompetenz von angehenden DaF-Lehrkräften im Rahmen eines deutschsprachigen Grundstudiums an?

-Welches unterrichtspraktische Modell kann hierbei eingesetzt werden? Welche Komponenten umfasst dieses Modell?

-Wie können die Lerninhalte der Deutschlehrerausbildung in der Türkei in dieses Modell integriert werden?

\section{Akademische Textkompetenz: Was steckt hinter diesem Begriff?}

Die Entwicklung des Begriffs akademische Textkompetenz ist auf die in den 60er Jahren begonnenen Literacy-Studien in der englischsprachigen Feldforschung zurückzuführen (Thonhauser 2007: 17). Diese Art von Forschung zielt darauf ab, die Rolle und Funktion von Schriftkundigkeit in einer Wissensgesellschaft und deren Einfluss auf die Möglichkeiten eines Individuums zur Partizipation am sozialen, politischen, ökonomischen und kulturellen Leben zu ermitteln (Sieber 2005: 396). In diesem Kontext wird die akademische Textkompetenz als eine Kompetenz definiert, welche die Teilhabe am Diskurs einer Disziplin und die Entwicklung der akademischen Literalität gewährleistet (Ballweg u.a. 2016: 137). Portmann-Tselikas und Schmölzer Eibinger definieren diese Kompetenz wie folgt:

\footnotetext{
Der erste Teil des Kompositums „Textkompetenz“ bezeichnet einen Gegenstandbereichs: Es geht um Texte, um Textuelles, um Textualität - und damit um etwas, was dem Bereich der Schriftlichkeit nahesteht, auch wenn es sich nicht immer mit ihm deckt. Der zweite Teil - Kompetenz - bringt es auf den Punkt, worum es hier hauptsächlich geht: Es ist die individuelle Fähigkeit, Texte lesen, schreiben und zum Lernen nutzen zu können. (2008: 5)
}

Die akademische Textkompetenz lässt sich zusammenfassend als eine Schlüsselkompetenz des Lernens definieren, mit der unterschiedliche Texte als Grundlage und Medium des Lernens genutzt werden können (vgl. Feilke 2019: 2; Schmölzer-Eibinger 2013: 30).

\section{Bedeutung und Rolle der Förderung der akademischen Textkompetenz von angehenden DaF-Lehrkräften}

Bei der Betrachtung des aktuellen Curriculums der Deutschlehrerausbildung zeigt sich, dass die fachbezogenen Studienfächer mit deutscher Unterrichtssprache den Kernbereich dieses Studienganges bilden (siehe Deregözü 2020: 155). Hinsichtlich der Strukturierung dieser fachbezogenen Studienfächer innerhalb des Grundstudiums wird ersichtlich, dass man sich in den ersten zwei Semestern zunächst darum bemüht, die 
fremdsprachlichen Kompetenzen der Studierenden mit kommunikativ orientierten Aufgaben im Rahmen sprachpraktischer Studienfächer (wie z. B. Sprechfertigkeit I \& II, Schreibfertigkeit I \& II, Lesefertigkeit I \& II etc.) zu fördern. Ab dem dritten Semester werden weitgehend kaum weitere sprachpraktische Studienfächer, sondern maßgeblich fachbezogene Studienfächer angeboten, welche auf das Aufbauen eines fachdidaktischen und berufspraktischen Wissens abzielen (wie z.B. Deutsche Literatur I, Lern- und Lehrmethoden des Deutschunterrichts, Sprachwissenschaft I etc.) (siehe YÖK 2018). Angesichts der Strukturierung der fachbezogenen Studienfächer lässt sich sagen, dass die Studierenden nach dem Aufbau ihrer alltagsbezogenen Kommunikationskompetenzen in den deutschsprachigen Fachunterricht entlassen werden. Gegenwärtig liegt somit ein Konzept vor, welches sprachpraktische Studienfächer und das Fachstudium voneinander trennt. Denn während in den sprachpraktischen Studienfächern hauptsächlich mit kommunikativen Aufgaben dialogisch, kreativ-assoziativ und fehlertolerant gearbeitet wird und es primär um das sprachliche Handeln in Situationen des zielsprachlichen Alltags geht, müssen die Studierenden in den fachbezogenen Fächern unter Bezugnahme zu einer fachinhärenten Methodik mit deutschsprachigen Fachtexten umgehen und Aufgaben systemkonform lösen können (vgl. Gladitz u.a. 2015: 155). Diese fehlende Verzahnung zwischen den Inhalten der sprachpraktischen und fachbezogenen Studienfächer birgt jedoch eine gewisse Problematik. So wird häufig beobachtet, dass viele der Studierenden trotz eines hochmotivierten Einstiegs in das fremdsprachliche Studium und deutlichen Erfolgen innerhalb der alltagsbezogenen Sprachbeherrschung (BICS), mit dem Beginn des deutschsprachigen Fachunterrichts mit sprachlichen Schwächen im kognitivakademischen Bereich (CALP) zu kämpfen haben (vgl. Balc1 1996: 65; Fandrych 2007: 283; Göpferich 2016: 280; Hatipoğlu 2015: 70; Schmölzer-Eibinger 2007: 207). Insbesondere zeigt sich, dass es den Studierenden an einer genügend ausgebildeten akademischen Textkompetenz fehlt, mit denen sie sich den schriftlichen Anforderungen des Lehramtsstudiums stellen können (ebd.). Folglich wird die akademische Textkompetenz lediglich im Kontext von Prüfungen unter Druck praktiziert und nicht als ein Medium des eigenständigen Lernens verstanden (vgl. Bohle 2016: 36; Dittmann u.a. 2003: 160). Die implizite Annahme, dass sich eine akademische Textkompetenz im Laufe des Studiums nebenbei ohne eine gezielte Förderung mit erworben lässt, führt letztendlich zu einer Beeinträchtigung der kognitiven und intellektuellen Entwicklung der Studierenden und der Eintritt in die Welt der Wissenschaft bleibt versperrt (vgl. Portmann-Tselikas/ Schmölzer-Eibinger 2008: 16).

\section{Die Integration des Wahlfachseminars „Akademisches Deutsch“ in das aktuelle Curriculum der Deutschlehrerausbildung in der Türkei}

Bei der Betrachtung der im Mai 2018 seitens des türkischen Hochschulrates durchgeführten Curriculumsrevision, zeigt sich im Hinblick auf die fachbezogenen Studienfächer der Deutschlehrerausbildung, dass die zuvor beschriebene Anordnung im Wesentlichen weiterhin besteht. Jedoch wurde in das aktuelle Wahlfachpool der Deutschlehrerausbildung das Seminar Akademisches Deutsch mit eingefügt. Die Ziele 
dieses neuen Seminars lauten unter anderem wie folgt: Einführung der angehenden DaF-Lehrkräfte in die Textsorten der DaF-Didaktik-Methodik und deren Eigenschaften; Betrachtung und Anwendung von unterschiedlichen Lese- und Schreibtechniken; Entwicklung der sprachlichen Kompetenzen für akademische Zwecke; Vermittlung der gezielten Planung des akademischen Textproduktionsprozesses (s. YÖK 2018). Angesichts dieser Zielsetzungen wird ersichtlich, dass dieses neue Seminar die besten Voraussetzungen für die gezielte Förderung der akademischen Textkompetenz bietet. Da es sich allerdings um ein noch recht neues Seminar handelt, fehlen konkrete Beispiele für die Unterrichtspraxis.

\section{Methodisch-didaktische Vorgehensweisen zur Förderung der akademischen Textkompetenz: Traditionelle und neue Ansätze}

Rückblickend herrschte bis Anfang der 1970er Jahre die Überzeugung vor, dass die Produktion eines Textes aus drei linear nacheinander ablaufenden Teilschritten Planen, Niederschreiben und Verbessern besteht und das Lesen und Schreiben gesondert zu fördernde Fähigkeiten bilden (vgl. Merz-Grötsch 2014: 52). Die Aufgabe der damaligen Schreibdidaktik bestand darin, die Merkmale guter Texte zu erfassen und ihr Fokus lag auf der Bewertung des abgeschlossenen Textproduktes (ebd.). Unter der Einflussnahme kognitiver Wissenschaften und der kognitiven Psychologie wurden große Anstrengungen unternommen, um etwas Licht in die Verarbeitung von Erfahrungen, Eindrücken und Informationen $\mathrm{zu}$ bringen und Fragen zur genaueren Konzeptualisierung der akademischen Textkompetenz gewannen immer mehr an Gewicht (siehe Flower/ Hayes 1981; Hayes 1996; Ludwig 1983; Spivey 1990). Denn es stellte sich heraus, dass man mit den herkömmlichen Bewertungskriterien, bei denen die Grammatik- und Rechtschreibfehler höher bewertet werden als der Inhalt, die Komplexität und Kohärenz eines Textes und mit den daraus folgenden Unterrichtskonzepten, bei denen lediglich das Endprodukt der Textproduktion und dessen formale Korrektheit ins Zentrum gestellt wird, nicht weit gekommen war und es auch nicht werden wird (Antos 1988: 37). Im Hinblick auf eine zeitgemäße Förderung entstand der Bedarf, die Lernenden unter Beachtung der Textproduktionsphasen gezielt darin zu fördern, eine aktiv betriebene Textproduktion durchzuführen. Als Ergebnis dieser Forschungen entwickelte sich das sog. Unterrichtsprinzip der Prozessorientierung.

\section{Das Unterrichtsprinzip der Prozessorientierung}

Bei der Prozessorientierung handelt es sich um ein Unterrichtsprinzip der zeitgenössischen Literalitätsdidaktik, welches sich auf das poetische Lehren und Lernen des Verfassens von Texten bezieht (vgl. Sieber 2005; Witschel 2017). Die Prozessorientierung darf jedoch nicht nur als eine weitere neue Technik betrachtet werden, welche didaktisch-methodisch auf eine Menge von Anweisungen zu reduzieren ist (Feilke 1988: 65). Dieses Unterrichtsprinzip beruht auf der Annahme, dass der 
Textproduktionsprozess einen Problemlöseprozess darstellt, der lediglich durch das komplexe Zusammenwirken unterschiedlicher kognitiver Phasen gelöst werden kann (vgl. Kruse/ Ruhrmann 2006: 13). Demgemäß wird im Rahmen eines entsprechenden Unterrichts der Prozesscharakter der Textproduktion als eine sequenzielle Anlage zur Durchführung von unterschiedlichen Übungsformen und Arbeitstechniken genutzt (vgl. Thonhauser 2010: 1036). Diese Übungsformen und Arbeitstechniken sind als ein „Koffer mit Werkzeugen, mit Techniken und Strategien“ zu verstehen, welcher den Lernenden gemeinsam mit dem Wissen, wie diese Werkzeuge laufend neu aufeinander abzustimmen sind, an die Hand gegeben werden muss (Kruse/ Perrin 2002: 10). Texte produzieren $\mathrm{zu}$ lernen heißt also, aus vielfältigen Werkzeugen, Techniken und Strategien auswählen und experimentieren zu dürfen, um für sich herauszufinden, welche dabei helfen, die individuelle Textproduktionen erfolgreich zu gestalten (vgl. Merz-Grötsch 2014: 68).

\section{Die Förderung der akademischen Textkompetenz nach dem 3-Phasen-Modell}

Mit der Kritik an dem Fehlen von Unterrichtskonzepten, welche eine unterrichtspraktische Umsetzungsmöglichkeit der Prozessorientierung darlegen, entwickelt Schmölzer-Eibinger auf Grundlage empirischer Arbeitsergebnisse das sog. 3Phasen-Modell zur Förderung der Textkompetenz. Das zentrale Ziel dieses Modells besteht darin, grundlegende literale Praktiken und Strategien im rezeptiven und produktiven Umgang mit Texten zu schulen, welche unabhängig von der Komplexität der Texte, den textsortenspezifischen Merkmalen und den Inhalten der Texte beherrscht werden müssen, um anhand von Texten lernen und in einer textgeprägten Sprache kommunizieren und lernen zu können (vgl. Schmölzer-Eibinger 2007: 215). SchmölzerEibinger charakterisiert (2008: 33) das 3-Phasen-Modell zur Förderung der Textkompetenz als ein lernerorientiertes, vielseitiges, einfach handzuhabendes und in unterschiedlichsten Lernkontexten verwendbares didaktisches Modell. Das Modell umfasst drei systematisch aufeinander bezogene und miteinander vernetzte Komponenten (siehe Tab.1).

\begin{tabular}{|c|c|c|}
\hline & Phase & Inhaltserläuterung \\
\hline \multirow[t]{2}{*}{1.} & Wissensaktivierung & $\begin{array}{l}\text { - Aktivierung des Vorwissens, der Erfahrungen, } \\
\text { Kenntnisse oder von spontanen Gedanken }\end{array}$ \\
\hline & & Textkonstruktion \\
\hline \multirow[t]{2}{*}{2.} & Arbeit an Texten & Textrekonstruktion \\
\hline & & Textfokussierung \& Textexpansion \\
\hline 3. & Texttransformation & $\begin{array}{l}\text { - Nutzen der vorhandenen Textinhalte- bzw.- } \\
\text { bedeutungen für das Schreiben eines Textes mit einer } \\
\text { anderen Textfunktion }\end{array}$ \\
\hline
\end{tabular}

Tab. 1: Das 3-Phasen-Modell zur Förderung der Textkompetenz (nach Schmölzer-Eibinger 2007) 
Die Phase der Wissensaktivierung zielt darauf ab, die Gedanken, Assoziationen, spontane Ideen und die vorhandenen Erfahrungen und Kenntnisse der Lernenden zu einem bestimmten Thema aufzurufen und für die Arbeit an einem Text verfügbar zu machen (vgl. Schmölzer-Eibinger 2008: 29ff.). Die zweite Phase Arbeit an den Texten bildet den Schlüsselbereich des 3-Phasen-Modells. Im Wesentlichen zielen die Aufgabenarrangements dieser Phase darauf ab, die Lernenden dazu anzuregen, Texte aus unterschiedlichen Perspektiven wahrzunehmen und in verschiedenen Kontexten zu reflektieren, $\mathrm{zu}$ rekonstituieren, $\mathrm{zu}$ überarbeiten oder neu $\mathrm{zu}$ konstituieren. Hierbei erfolgt die Arbeit an den Texten in den drei Stufen Textkonstruktion, Textrekonstruktion, Textfokussierung \& Textexpansion (ebd.):

1. Stufe - Textkonstruktion: Innerhalb der ersten Stufe erhalten die Lernenden Textfragmente, die sie vervollständigen müssen. Bei diesen Textfragmenten kann es sich um einzelne Sätze, Absätze oder kurze Textpassagen handeln. Um die Textfragmente ergänzen zu können, sollen die Lernenden durch den gezielten Einsatz unterschiedlicher Aufgaben dazu aufgefordert werden, die vorhandenen Textteile immer wieder aufs Neue zu lesen, zu überprüfen, zu überarbeiten oder zu verbessern.

2. Stufe - Textrekonstruktion: In der zweiten Stufe der Textarbeit werden die Lernenden dazu aufgefordert, den Text, den sie gelesen haben, möglichst genau zu rekonstruieren. $\mathrm{Zu}$ diesem $\mathrm{Ziel}$ sollten Aufgaben angeboten werden, welche die Lernenden dazu auffordern, die Texte mehrfach und ggf. aus unterschiedlichen Perspektiven zu lesen, Sinnzusammenhänge zu überprüfen und zu verdeutlichen.

3. Stufe: Textfokussierung \& Textexpansion: Die im Rahmen der dritten Stufe eingesetzten Aufgabenarrangements sollen die Lernenden dazu auffordern, relevante Informationen in einem Text zu erkennen, miteinander zu verbinden und zu erweitern.

In der dritten Phase des 3-Phasen-Modells zur Förderung der Textkompetenz geht es darum, Texte aus ihren ursprünglichen Kontexten herauszulösen und in neue Kontexte zu transferieren (ebd.).

\section{Unterrichtspraktische Anwendungsbeispiele zur Förderung der akademischen Textkompetenz von angehenden DaF-Lehrkräften}

Im weiteren Verlauf werden unter Beachtung des oben beschriebenen Modells, unterrichtspraktische Beispiele präsentiert, welche im Rahmen des Seminars Akademisches Deutsch ${ }^{2}$ zur Förderung der akademischen Textkompetenz eingesetzt werden können. Die Zielgruppe dieser Unterrichtsbeispiele bilden Studierende eines Deutschlehrerausbildungsprogramms, dessen Unterrichtssprache Deutsch ist. Um den in der einschlägigen Literatur genannten Forderungen nachzukommen und um der Gefahr entgegen zu wirken, einen Förderunterricht durchzuführen, der fachliche Inhalte der Deutschlehrerausbildung ausblendet, wurden gezielt unterschiedliche Fachtexte in Form von Textsets als Arbeitsbasis herangezogen, die einen inhaltlichen Bezug zum Fachpensum der Deutschlehrerausbildung haben und deren fachliche Themeninhalte als

\footnotetext{
${ }^{2}$ Der Begriff Akademisches Deutsch legt eine Variante des akademischen Sprachgebrauchs (CALP) dar und bezeichnet im vorliegenden Zusammenhang das spezifische Sprachregister der akademischen Lehre und Forschung im Kontext der Deutschlehrerausbildung (vgl. Vollmer/ Thürmann 2010: 109).
} 
ein Ausgangspunkt für eine Diskussion genutzt werden können (vgl. Fandrych 2007: 283; Lupo u.a. 2017: 43). Es ist zu betonen, dass die in der Tabelle 2 dargelegten Themenbereiche sowohl als einzelne Module voneinander unabhängig, aber auch nacheinander bzw. in einer anderen Reihenfolge eingesetzt werden können (s. Tab. 2).

\begin{tabular}{|c|c|c|c|}
\hline Themenbereich: & Textnummer: & & Bestandteile der Textsets: \\
\hline \multirow{6}{*}{$\begin{array}{l}\text { a. Relevanz von } \\
\text { Lernstrategien im DaF- } \\
\text { Unterricht }\end{array}$} & \multirow{2}{*}{1} & Titel: & Lernstrategien $^{3}$ \\
\hline & & Textsorte: & Auszug aus einem Lehrbuch \\
\hline & \multirow[t]{2}{*}{2} & Titel: & $\begin{array}{l}\text { Warum soll man Lerntechniken } \\
\text { vermitteln? }^{4}\end{array}$ \\
\hline & & Textsorte: & Auszug aus einer Monographie \\
\hline & \multirow[t]{2}{*}{3} & Titel: & $\begin{array}{l}\text { Grenzen und Missverständnisse bei } \\
\text { Lernstrategien }^{5}\end{array}$ \\
\hline & & Textsorte: & Auszug aus einem Lehrbuch \\
\hline \multirow[t]{6}{*}{$\begin{array}{l}\text { b. Rolle von Medien } \\
\text { im DaF-Unterricht }\end{array}$} & \multirow[t]{2}{*}{1} & Titel: & $\begin{array}{l}\text { Lernmaterialien und Medien: Eine erste } \\
\text { Annäherung }^{6}\end{array}$ \\
\hline & & Textsorte: & Auszug aus einem Lehrbuch \\
\hline & \multirow[t]{2}{*}{2} & Titel: & $\begin{array}{l}\text { Medien im Fremdsprachenunterricht }- \text { Drei } \\
\text { DaF-Lehrer berichten über den } \\
\text { Medieneinsatz in ihrem eigenen } \\
\text { Unterricht }^{7}\end{array}$ \\
\hline & & Textsorte: & Fiktiver Interviewtext \\
\hline & \multirow[t]{2}{*}{3} & Titel: & $\begin{array}{l}\text { Die Funktion von Medien im Deutsch als } \\
\text { Fremd- und Deutsch als Zweitsprache- } \\
\text { Unterricht }{ }^{8}\end{array}$ \\
\hline & & Textsorte: & Auszug aus einem Handbuch \\
\hline c. Einsatz von & 1 & Titel: & Sozialformen: Überblick ${ }^{9}$ \\
\hline
\end{tabular}

${ }^{3}$ Ballweg, Sandra u.a. (2013): Lernstrategien. In: Wie lernt man die Fremdsprache Deutsch? 2. Deutsch Lehren Lernen. Hg. v. Sandra Ballweg, Sandra Drumm, Britta Hufeisen, Johanna Klippel, Lina Pilypaitytè. München: Goethe-Institut, 94-96.

4 Rampillon, Ute (1985): Warum soll man Lerntechniken vermitteln?. In: Lerntechniken im Fremdsprachenunterricht. Hg. v. Ute Rampillon. Forum Sprache. München: Hueber, 18-21.

5 Bimmel, Peter/ Rampillon, Ute (2000): Grenzen und Missverständnisse bei Lernstrategien. In: Lernerautonomie und Lernstrategien. Fernstudieneinheit 23. Hg. v. Peter Bimmel \& Ute Rampillon. München: Goethe-Institut, 60-61.

${ }^{6}$ Rösler, Dietmar/ Würffel, Nicola (2014): Lernmaterialien und Medien: Eine erste Annäherung. In: Lernmaterialien und Medien. Deutsch Lehren Lernen. Band 5. Hg. v. Dietmar Rösler \& Nicola Würffel.München:Klett-Langenscheidt, 12-14.

${ }^{7}$ Erstellt von den Verfasserinnen dieser Arbeit.

8 Rösler, Dietmar (2010): Die Funktion von Medien im Deutsch als Fremd- und Deutsch als Zweitsprache-Unterricht. In: Deutsch als Fremd- und Zweitsprache. Ein internationales Handbuch (1. Halbband). Hg. v. Hans-Jürgen Krumm, Christian Fandrych, Britta Hufeisen, Claudia Riemer. Berlin: De Gruyter Mouton, 1199-1200.

${ }^{9}$ Schwerdtfeger, Inge C. (2007): Sozialformen: Überblick. In: Handbuch Fremdsprachenunterricht. Hg. v. Karl-Richard Bausch, Herbet Christ, Hans-Jürgen Krumm. Tübingen: Narr Francke Attempto Verlag, 247-251. 


\begin{tabular}{|c|l|l|l|}
\hline \multirow{2}{*}{$\begin{array}{c}\text { verschiedenen } \\
\text { Sozialformen im DaF- } \\
\text { Unterricht }\end{array}$} & Textsorte: & Auszug aus einem Handbuch \\
\cline { 2 - 4 } & \multirow{2}{*}{2} & Titel: & Einsatz von Arbeits- und Sozialformen \\
\cline { 2 - 4 } & Textsorte: & Auszüge aus einem Lehrbuch \\
\cline { 2 - 4 } & \multirow{2}{*}{3} & Titel: & Die Sozialformen des Unterrichts ${ }^{11}$ \\
\cline { 3 - 4 } & Textsorte: & Zeitschriftenaufsatz \\
\hline
\end{tabular}

Tab. 2.: Bestandteile der Textsets

Darüber hinaus ist zu betonen, dass die in der Tabelle 2 dargelegten Themenbereiche auch durch andere Themenbereiche ausgetauscht werden können. Zu erwähnen ist auch, dass bei dem Einsatz der präsentierten Unterrichtsbeispiele die Moodle-Lernplattform genutzt wurde. Es ist jedoch auch möglich, die präsentierten Beispiele im gängigen Klassenunterricht einzusetzen.

\section{Typische Beispiele für Prozessorientierung in der Phase 1}

Im Folgenden werden unterschiedliche Beispiele aufgeführt, die im Rahmen der Wissensaktivierungsphase während der Unterrichtsdurchführung einsetzbar sind.

Assoziatives Sprechen über eine Ausgangsfrage: Zur Wissensaktivierung eignet es sich, den Unterricht mit einer kritischen Ausgangsfrage zu beginnen. So kann beispielweise zum Thema Rolle von Medien im DaF-Unterricht eine Abbildung entwickelt werden, in der die Ausgangsfrage „Garantiert der Einsatz von unterschiedlichen Medien einen besseren DaF-Unterricht?“ gemeinsam mit zwei unterschiedlichen Antworten abgebildet ist (s. Abb. 1).

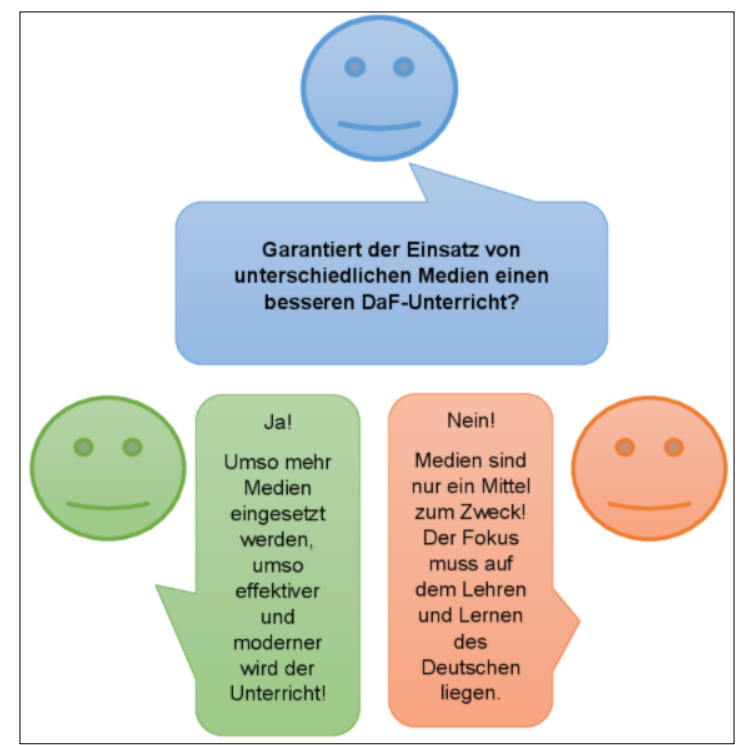

Abb. 1: Brainstorming in Anlehnung an eine Ausgangsfrage

${ }^{10}$ Funk, Hermann u.a. (2017): Lernmaterialien und Medien: Eine erste Annäherung. In: Aufgaben, Übungen, Interaktion. Deutsch lehren lernen. Band 4.Hg. v.Hermann Funk, Christina Kuhn, Dirk Skiba, Dorothea Spaniel-Weise, Rainer E. Wicke. München: Klett Langenscheidt, S. 57-58.

${ }^{11}$ Nuhn, Hans-Eberhard: Die Sozialformen des Unterrichts. In: Pädagogik 52 (2)(2000), 10-13. 
Nach der Betrachtung dieser Abbildung kann die Lehrkraft den Studierenden folgende Fragen stellen: „Wie lautet die Fragestellung?“, „Was denken Sie über die zwei Antworten zu dieser Fragestellung?" und „Wie würden Sie selbst auf diese Fragestellung antworten?“. Hierbei werden die Erfahrungen und Gedanken der Studierenden schrittweise aufgerufen und es entsteht ein Bewusstsein darüber, dass es kontroverse Standpunkte bezüglich dieses Themenbereichs gibt.

Vertextung von Darstellungsformen: Eine weitere Möglichkeit das Wissen der Studierenden $\mathrm{zu}$ aktivieren, besteht auch in der Vertextung einer Darstellungsform (siehe Leisen 2013: 252). In Bezug auf den Themenbereich Einsatz verschiedener Sozialformen für den DaF-Unterricht wurde beispielsweise eine Darstellungsform entwickelt, welche die vier Sozialformen des Unterrichts visualisiert (siehe Abb. 2).

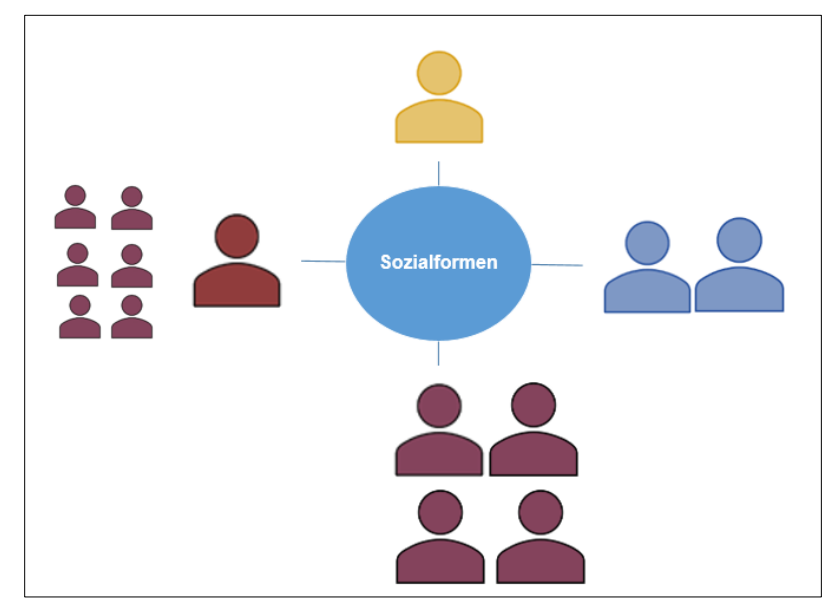

Abb. 2: Visualisierung der Sozialformen

Wie aus der Abbildung zu entnehmen ist, werden die Sozialformen Einzelarbeit, Partnerarbeit, Gruppenarbeit und Frontalunterricht visualisiert. Während der Präsentation dieser Abbildung fragt die Lehrkraft die Studierenden, was sie mit dem Begriff Sozialform assoziieren. Nach der mündlichen Formulierung der ersten Vermutungen und Gedanken wird an die Studierenden ein Arbeitsblatt verteilt, welches eine allgemeine Definition des Begriffs Sozialformen und die Bezeichnungen der einzelnen Sozialformen enthält. Im ersten Schritt werden die Studierenden dazu aufgefordert, die vorliegenden Bezeichnungen den abgebildeten SozialformenAbbildungen zuzuordnen (siehe Abb. 3). 


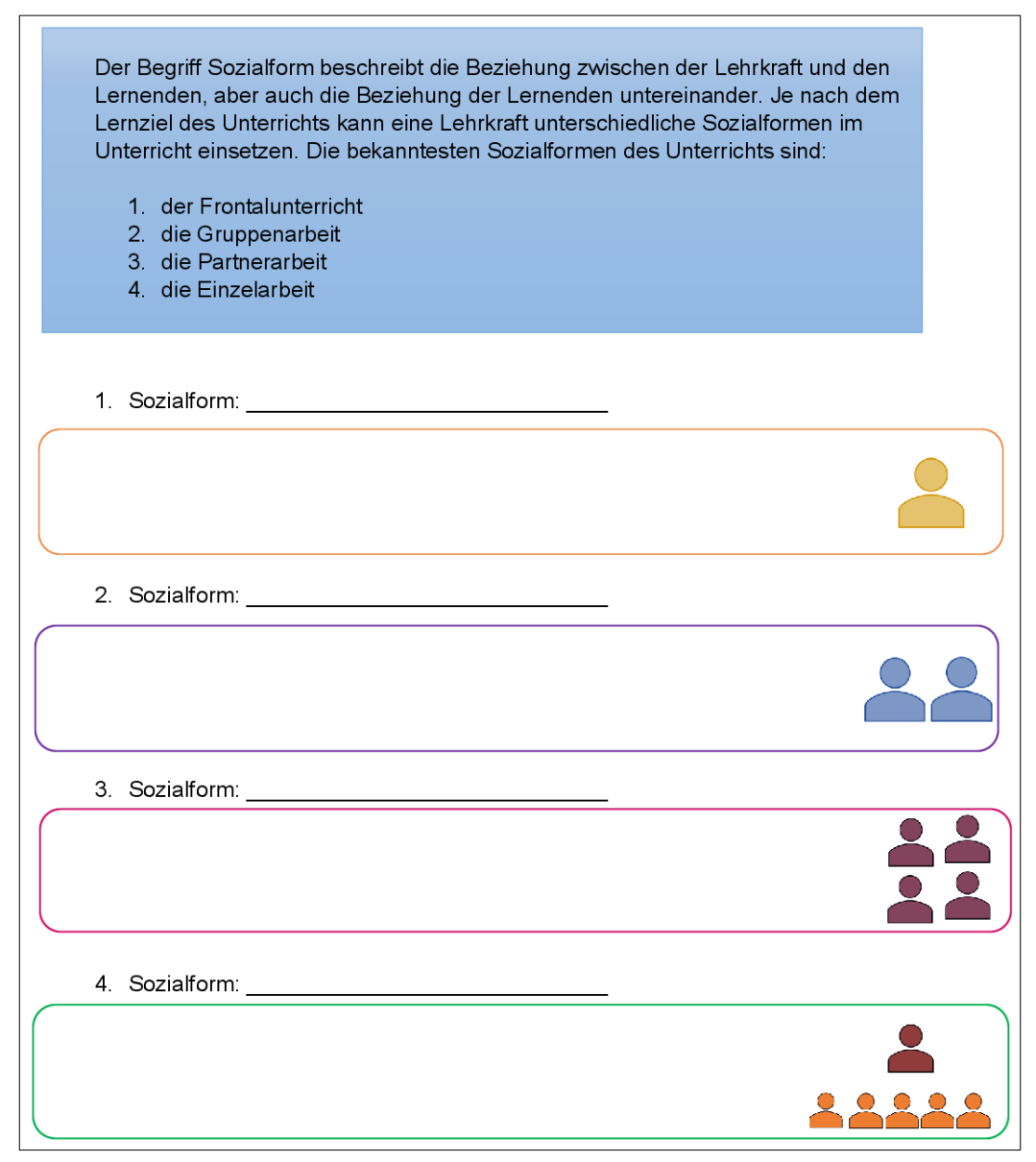

Abb. 3: Vertextung der Sozialformen

Nach der Zuordnung der Bezeichnungen werden die Studierenden dazu aufgefordert, die vorliegenden Abbildungen mit ihren eigenen Worten zu verschriftlichen.

\section{Typische Beispiele für Prozessorientierung in der Phase 2}

Im weiteren Verlauf werden die entwickelten Beispiele entsprechend der drei Schritte der zweiten Textarbeitsphase präsentiert (siehe Tab. 1).

\section{Schritt 1: Textkonstruktion}

Im ersten Schritt der Textarbeitsphase können die Studierenden mit folgenden Beispielen zur konstruktiven Arbeit mit unterschiedlichen Textfragmenten angeregt werden:

Ergänzung von fehlenden Satzteilen: Zur Realisierung des ersten Schrittes der Textarbeit können den Studierenden Textfragmente aus den zu bearbeitenden Texten mit fehlenden Satzteilen vorgelegt werden (siehe Abb. 4). Beim Vervollständigen der Textfragmente betreten die Studierenden ein thematisches Neuland und die Kreativität 
und Phantasie wird stärker gesteigert, da die Assoziationen und Gedanken der Studierenden mit in die Textkonstruktionsphase einfließen (vgl. Schmölzer-Eibinger 2013: 30). So können den Studierenden z.B. drei kurze Textfragmente aus dem zweiten Textset vorgelegt und folgende Aufforderung gestellt werden: „In den präsentierten Textfragmenten fehlen ganze Satzteile. Versuchen Sie die fehlenden Satzteile mit ihren eigenen Worten zu ergänzen!“‘.

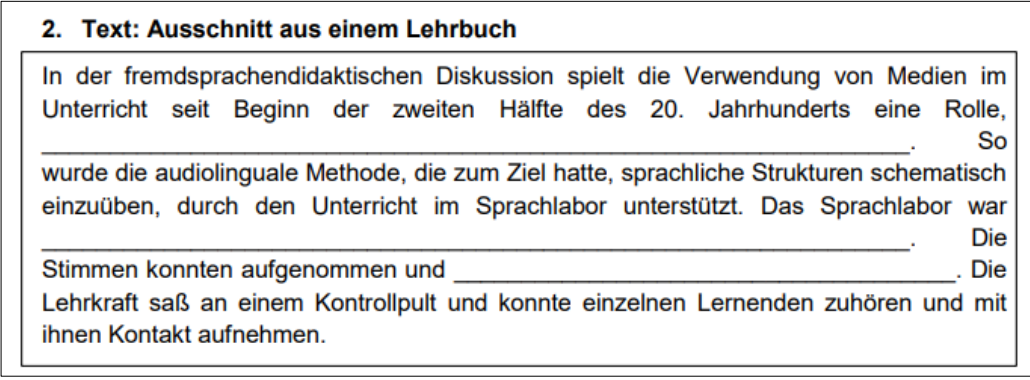

Abb. 4: Ergänzung von fehlenden Satzteilen

Nachdem die Studierenden die fehlenden Satzteile in den drei Textfragmenten mit ihren eigenen Worten ergänzt haben, werden diese im Plenum diskutierend miteinander und mit dem Originaltext verglichen.

Zuordnung von Zwischenüberschriften: Eine weitere Form der Textkonstruktion kann auch durch die Zuordnung von vorgelegten Zwischenüberschriften durchgeführt werden (siehe Leisen 2013: 210). Dementsprechend kann die Lehrkraft den Studierenden Textabschnitte aus den jeweils zu bearbeitenden Textsets vorlegen und sie dazu auffordern, diese mit den folgenden Zwischenüberschriften $\mathrm{zu}$ beschriften: Allgemeine Informationen, Definition „X“, Nennung von Vorteilen und Nennung von Nachteilen (siehe Abb. 5).

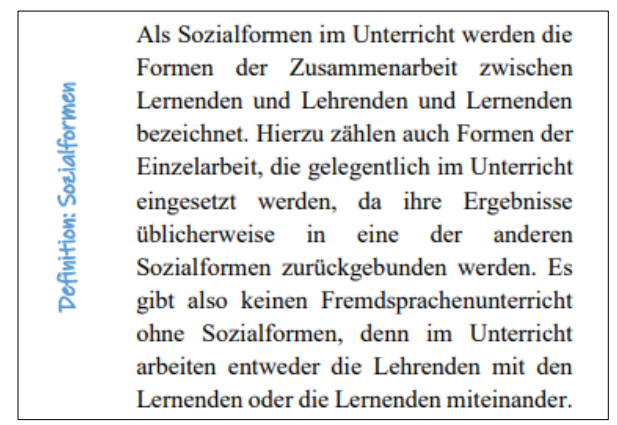

Abb.5: Beschriften von Textabschnitten

\section{Schritt 2: Textrekonstruktion}

Folgende unterrichtspraktische Beispiele können für die Rekonstruktion der bearbeiteten Texte herangezogen werden:

Suchen von Sätzen: Das Suchen nach Sätzen hat zum Ziel, dass die Studierenden aus einer Auswahl von Sätzen den inhaltlich am besten passenden Satz aussuchen (siehe Abb. 6). Die Durchführung dieser Übung fördert das Verstehen inhaltlicher Details, die 
über das Globalverstehen und das Rekonstruieren der Textstruktur hinausgehen (Leisen 2013: 208). In Bezug auf den Themenbereich Rolle von Medien im DaF-Unterricht können den Studierenden, welche zuvor mit Textfragmenten gearbeitet haben, die Originaltexte ausgehändigt werden, in denen jeweils ein ganzer Satz fehlt. Zur Textrekonstruktion müssen die Studierenden die Texte und Sätze mehrmals lesen und eine Auswahl treffen.

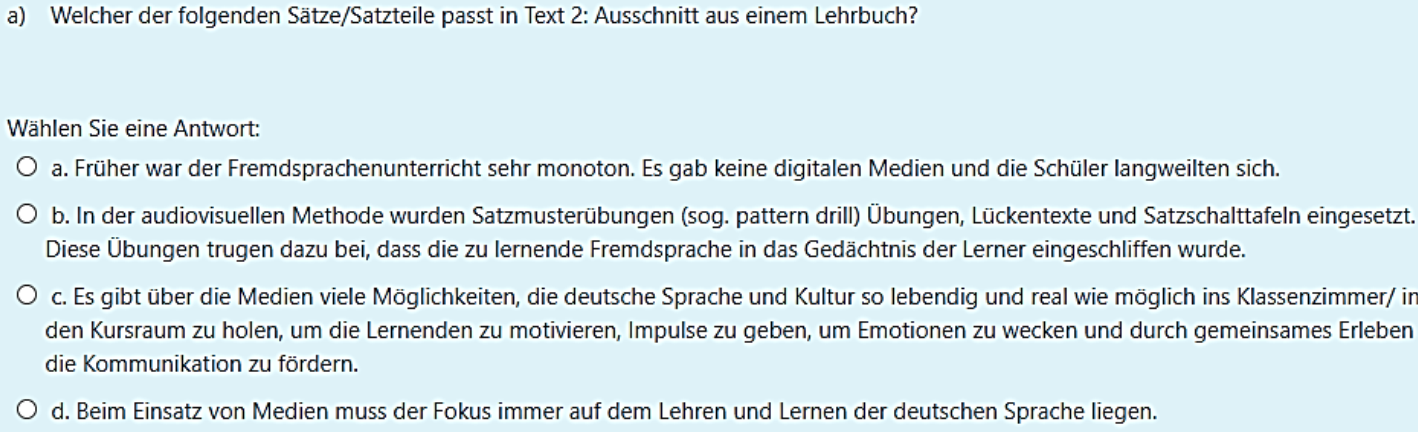

Abb. 6. Ausschnitt zur Satzsuche

Strukturierung von Texten: Eine weitere Möglichkeit zur Durchführung der Textrekonstruktion besteht auch in der Aushändigung von Texten, die von ihrer Gestaltung her nicht verständnisfördernd aufgebaut sind (siehe Leisen 2013: 146). Mit dem Ziel die Studierenden in der Kategorisierung und Abstraktion textlicher Inhalte zu schulen, können die zu bearbeitenden Originaltexte, ohne eine inhaltliche Veränderung durchzuführen, gezielt ohne Absätze und Zwischenüberschriften abgetippt und in dieser Form an die Studierenden ausgehändigt werden. In der Unterrichtsdurchführung kann folgende Aufgabenstellung formuliert werden: „Die Originaltexte sind leider ohne Absätze geschrieben. Machen Sie diese Texte lesefreundlicher. Das hilft Ihnen selbst, die Texte besser zu verstehen. Teilen Sie hierfür den Text in physikalisch sinnvolle Abschnitte ein und notieren Sie zu jedem Abschnitt eine kurze Überschrift!“‘

\section{Schritt 3: Textfokussierung und -expansion}

Zur Durchführung der dritten Stufe der Textarbeitsphase können folgenden Beispiele zum Einsatz kommen:

Farborientiertes Markieren: Der dritte Schritt der Textarbeitsphase kann mit dem farborientierten Markieren beginnen. Der Zweck dieser Aufgabe liegt darin, dass sich die Studierenden mehrfach mit einem oder mehreren Text(en) konzentriert auseinandersetzen müssen (vgl. Leisen 2013: 150). So können die Studierenden z.B. bezüglich des Themenbereichs Rolle von Medien im DaF-Unterricht folgendermaßen zum farborientierten Markieren aufgefordert werden: „a) Welche Medien werden in diesen Texten genannt? Markieren Sie alle genannten Medien mit der Farbe Blau!; b) Unterstreichen Sie alle wichtige Textstellen mit der Farbe Gelb!; c) Welche Vorteile bzw. Nachteile werden zum Medieneinsatz genannt? Markieren Sie die Vorteile grün und die Nachtteile rot!“. Die Idee des farborientierten Markierens besteht darin, eine 
zyklische Bearbeitung der Texte einzuleiten, durch die eine Fokussierung auf die relevanten Informationen gefördert wird. Die durch die farblichen Markierungen hervorgehobenen Textstellen sollten als Anker für die fortsetzende Textarbeit dienen (ebd.).

Exzerpt-Tabelle: Für die Durchführung der Textexpansion kann an die Studierenden eine vorgefertigte Exzerpt-Tabelle verteilt werden (siehe Abb. 7).

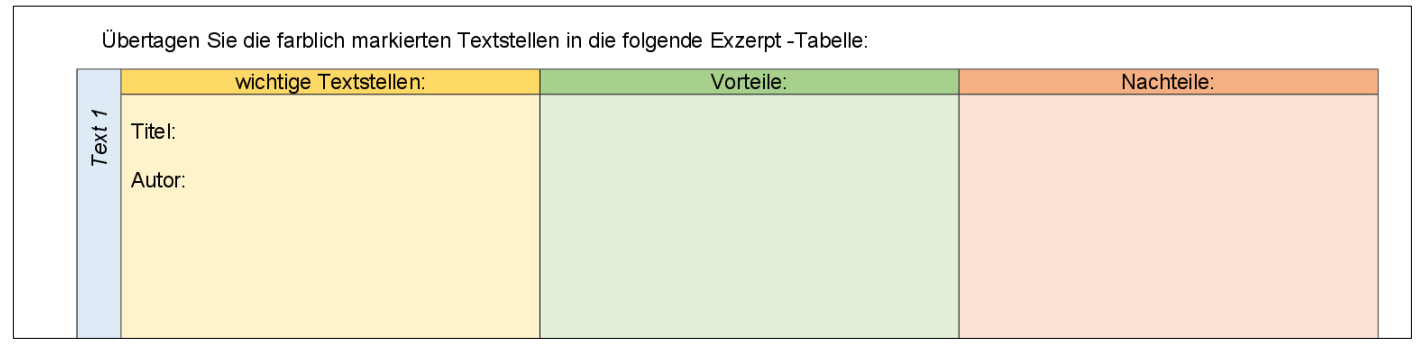

Abb. 7: Ausschnitt aus der Exzerpt-Tabelle

Diese Exzerpt-Tabelle enthält für jeden zu bearbeitenden Text des zweiten Textsets jeweils einen eigenen Tabellenabschnitt. Darüber hinaus können in dieser ExzerptTabelle z.B. auch dieselben Farben wie aus der vorherigen Textbearbeitungsphase wieder mit aufgegriffen werden. Demgemäß werden die Studierenden dazu aufgefordert, die aus der farborientierten Markierungsarbeit gewonnenen Inhalte in diese Exzerpt-Tabelle zu übertragen und diese Inhalte mit eigenen Bemerkungen, Notizen und eigenen Ideen zu erweitern. Diese aktive Auseinandersetzung mit den Texten trägt dazu bei, dass sich die Studierenden einen Daten-Pool für die darauffolgende Texttransformationsphase anlegen (vgl. Merz-Grötsch 2014: 81).

Ordnen von Aussagen entsprechend ihrer Wichtigkeit: Nach dem Ausfüllen der Exzerpt-Tabellen können im Rahmen des dritten Schrittes der Textarbeitsphase die Studierenden z.B. auch dazu aufgefordert werden, die ihrerseits ausgefüllten ExzerptTabellen zu betrachten und ein weiteres Arbeitsblatt zu bearbeiten, bei dem es darum geht, die in der Tabelle enthaltenen Aussagen bzw. Argumente entsprechend ihrer Wichtigkeit $\mathrm{zu}$ nummerieren (siehe Abb. 8). Hierbei kann folgende Aufgabenformulierung zum Einsatz kommen: ,a) Welche wichtigen Aussagen, Vorteile und Nachteile aus der Exzerpt-Tabelle finden Sie persönlich wichtig? Kreuzen Sie diese an!; b) Wählen Sie sechs Aussagen, Vorteile und Nachteile aus und übertragen Sie diese in das Arbeitsblatt!; c) Nummerieren Sie diese sechs ausgewählten Aussagen, Vorteile und Nachteile entsprechend ihrer Wichtigkeit!“. 


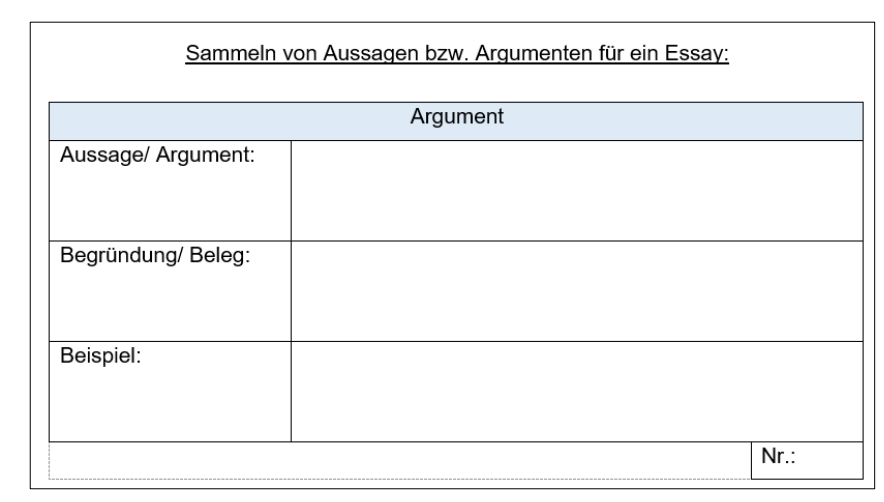

Abb. 8: Sammeln und Ordnen von Aussagen und Argumenten

Durch die Arbeit an dem Arbeitsblatt werden die Studierenden dazu aufgefordert, die Informationen aus der Exzerpt-Tabelle nochmals zu sichten und persönlich zu bewerten. Wie aus der Abbildung $8 \mathrm{zu}$ entnehmen ist, werden die Studierenden somit gezielt darin geschult, Argumente bzw. wichtige Aussagen gemeinsam mit Begründungen und Beispielen festzuhalten (siehe Merz-Grötsch 2014: 216).

\section{Typische Beispiele für Prozessorientierung in der Phase 3}

In der dritten Phase der Textproduktion geht es darum, das durch die rekonstruktive und produktive Textbearbeitung entstandene Wissen für die Produktion eines eigenen Textes zu transferieren (vgl. Schmölzer-Eibinger 2008: 33). Es ist zu betonen, dass sich für die Durchführung der Texttransformationsphase im universitären Kontext insbesondere das Verfassen eines Essays eignet, da es eine effiziente Auseinandersetzung mit mehreren Texten zu einem Thema voraussetzt (siehe Stadter 2003: 65ff.). An dieser Stelle ist zu betonen, dass bereits am Anfang des Kursbeginns eine kurze Erarbeitungsphase bezüglich der Textsorte Essay und dessen Eigenschaften durchgeführt werden sollte. Demgemäß kann z.B. in Bezug auf den Themenbereich Rolle von Medien im DaF-Unterricht folgende Essayfrage formuliert werden: „Im DaFUnterricht können viele verschiedene digitale Medien zur Förderung des Lehrens und Lernens der deutschen Sprache eingesetzt werden. Allerdings ist ihr Einsatz sehr umstritten. Halten Sie den Einsatz von digitalen Medien im DaF-Unterricht für sinnvoll?“، Trotz einer gemeinsamen und ausgiebigen Vorbereitung auf die Texttransformationen bewährt es sich, auch innerhalb der Texttransformationsphase die Studierenden durch unterschiedliche Aufgabenarrangements zu unterstützen.

Das Schreiben nach einer Gliederung: Zur Unterstützung der Texttransformationsphase kann den Studierenden eine bereits vorgefertigte Word-Datei vorgelegt werden, die den typischen Aufbau eines Essays in Form eines farblichen Gliederungsrasters wiedergibt (siehe Abb. 9). 


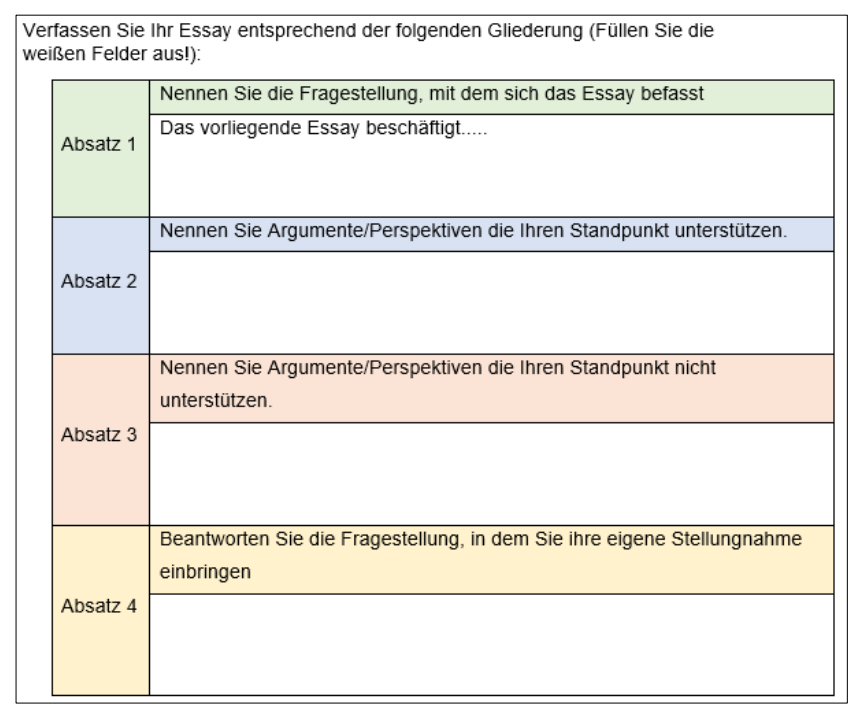

Abb. 9: Gliederungsraster zum Verfassen eines Essays

Diese Strukturgebung hat eine erfolgabsichernde Funktion, da mit ihr der erwartete Textaufbau und -umfang vorgelegt wird (vgl. Leisen 2013: 182). Außerdem können die anhand dieser Gliederung entstandenen Textprodukte auch leichter miteinander verglichen und überarbeitet werden.

Durchführung einer virtuellen Schreibkonferenz: Eine weitere effiziente Möglichkeit zur Unterstützung der Texttransformationsphase besteht in der Durchführung einer virtuellen Schreibkonferenz (siehe Merz-Grötsch 2014: 196ff.). Hierbei kann die Lehrkraft die eingereichten Essays der Studierenden anonymisieren und gemeinsam mit einem vorgefertigten Bewertungsbogen an einen anderen Studierenden weiterleiten. Nachdem die Studierenden die erhaltenen Essays bewertet haben, schicken sie diese an die Lehrkraft zurück, welche wiederum diese zur Revision wieder an die eigentlichen Verfasser leitet.

Abschließend ist zu betonen, dass bei der genaueren Betrachtung des 3-PhasenModells schnell deutlich wird, dass der Prozesscharakter der Textproduktion als eine sequenzielle Anlage zur Unterrichtsdurchführung dient und dass durch das gezielte Ineinandergreifen der drei Phasen das Prinzip der Prozessorientierung gewährleistet wird. Durch die gezielte Wissensaktivierung in der ersten Phase entsteht eine Ausgangsgrundlage für die darauffolgende Textarbeitsphase. Die in der zweiten Phase kleinschrittig vorangetriebene rekonstruktiv-produktive Textbearbeitung bildet wiederum einen Impuls für die Neukonzeption von Texten im Rahmen der dritten Phase. Durch das Zusammenwirken der einzelnen Funktionen dieses Modells wird folglich ein Mechanismus der Textarbeit in Gang gesetzt, durch die jene kognitiven Prozesse angeregt und intensiviert werden, die im Umgang mit Texten besonders produktivitätssteigernd und lernwirksam sind (vgl. Schmölzer-Eibinger 2008: 33). 


\section{Schlussbetrachtung und Vorschläge für Deutschlehrerausbildungsprogramme in der Türkei}

Die akademische Textkompetenz hat eine epistemische, also wissenskonstruierende Funktion, welche die Qualität der Lehrerausbildung maßgeblich beeinflusst (vgl. Göpferich 2016: 277). Trotz dieser hohen Bedeutung, zeigt die gegenwärtige Unterrichtspraxis an den lehrerausbildenden Studiengängen, dass die akademische Textkompetenz, also die Fähigkeit sich in der Welt der Texte zu orientieren und die eigenen sprachlichen Ressourcen als ein Werkzeug des Denkens, Kommunizierens und Lernens $\mathrm{zu}$ nutzen, im Unterricht nicht systematisch aufgebaut, sondern vielmehr einfach vorausgesetzt wird (vgl. Portmann- Tselikas/ Schmözler-Eibinger 2008: 14). Es gilt jedoch, dass ,Know-How ‘ der Textproduktion durch das Einbringen von didaktisch begründeten Unterrichtseinheiten für die universitäre Unterrichtspraxis verfügbar zu machen. Mit dem Ziel, die Studierenden gezielt auf die fachlichen Inhalte der Deutschlehrerausbildung vorzubereiten, ist insbesondere für eine inhaltliche Umorientierung innerhalb sprachpraktischer Studienfächer zu plädieren. Denn je mehr Studierende Texte entsprechend des Unterrichtsprinzips der Prozessorientierung bearbeiten, d.h. gezielt erfassen, durchdringen, rezipieren und reproduzieren, umso mehr lernen sie, sich den Kriterien der Textualität zu nähern und ein höherstufiges Niveau ihrer akademischen Textkompetenz zu erreichen (Janich, 2008: 18ff.). Ausgehend von der Forderung zur Entwicklung von konkreten methodisch und didaktischen Hilfestellungen, welche auf die jeweils vorliegende Problematik zugeschnitten, praxisnah und direkt einsetzbar sind, zielt die vorliegende Arbeit mit der Darlegung der präsentierten Unterrichtsbeispiele darauf ab, einen konkreten Beitrag zur Förderung der akademischen Textkompetenz von angehenden DaF-Lehrkräften zu leisten. Es ist $\mathrm{zu}$ betonen, dass das Spektrum zur Förderung der akademischen Textkompetenz breit gefächert ist und dass hier nur ein kurzer Einblick in die Umsetzungsmöglichkeiten der Prozessorientierung gegeben werden konnte.

\section{Literaturverzeichnis}

Antos, Gerd (1988): Eigene Texte herstellen! Argumente aus der Sicht der Schreibforschung. Der Deutschunterricht 3, 37-47.

Balcı, Tahir (1996): Deutsch als Unterrichtssprache bei germanistischen Lehrveranstaltungen. ÖDaFMitteilungen 1, 65-70.

Ballweg, Sandra u.a. (2016): Schreiberfahrung von Studierenden aus verschiedenen Fächergruppen und deren wahrgenommener Unterstützungsbedarf beim akademischen Schreiben: Ergebnisse einer Querschnittsstudie. Schreibberatung und Schreibförderung: Impulse aus Theorie, Empirie und Praxis. Hg. v. Sandra Ballweg.Frankfurt am Main: Peter Lang, 137-172.

Bausch, Karl- Richard (1992): Plädoyer für eine wissenschaftlich begründete, professionalisierte sowie auf Mobilität ausgerichtete Fremdsprachenausbildung. Manuskripte zur Sprachlehrforschung: Die Ausbildung von Fremdsprachenlehrern als Gegenstand der Forschung 33, 23 - 30.

Bohle, Ulrike (2016): Schreiben als Lerngegenstand, Lehrmedium und Lernvorraussetzung. Zur Schreibdidaktik an Schulen und Hochschulen. Ballweg, Sandra (Hg.):Schreibberatung und 
Schreibförderung: Impulse aus Theorie, Empirie und Praxis. Frankfurt am Main: Peter Lang, 1743.

Cummins, Jim (1999): BICS and CALP: Clarifying the distinction. (Report No: ED 438 551). Washington D.C.: ERIC Clearinghouse on Languages and Linguistics.

Decker, Lena u.a. (2015): Modellierung und Erfassung akademischer Textkompetenzen. AkaTex Working Papers 3. 2. korrigierte Auflage. Siegen und Köln: Universität Siegen und Universität Köln.

Deregözü, Aysel (2020): Geçmişten Günümüze Almanca Öğretmenliği Lisans Programlarına İlişkin Karşılaştırmalı Bir Çalışma. Diyalog. Interkulturelle Zeitschrift für Germanistik Sonderausgabe: 85 Jahre Germanistik in der Türkei, 143 -161.

Dittmann, Jürgen u.a. (2003): Schreibprobleme im Studium- Eine empirische Untersuchung. Wissenschaftich schreiben-lehren und lernen. Hg. v. Konrad Ehlich und Angelika Steets. Berlin: De Gruyter, 155-185.

Fandrych, Christian (2007): Aufgeklärte Zweisprachigkeit als Ziel und Methode der Germanistik nichtdeutschsprachiger Länder. Schmölzer-Eibinger, Sabine/ Weidacher, Georg (Hg.): Textkompetenz. Tübingen: Gunter Narr, 275-298.

Feilke, Helmuth (2019): Bildungssprache. https://epub.ub.unimuenchen.de/61963/1/Feilke_Bildungssprache.pdf (letzter Zugriff: 20.12.2020).

Feilke, Helmuth (1988): Ordnung und Unordnung in argumentativen Texten. Zur Entwicklung der Fähigkeit, Texte zu strukturieren. Der Deutschunterricht 3, 65-81.

Flower, Linda/ Hayes, John R. (1981): A Cognitive Process Theory of Writing. College Composition and Communication 32(4), 365-387.

Franken, Anna U. (2020): „Wir hatten ja schon öfters mal Texte.“ - Schülervorstellungen von Texten und materialgestütztes Schreiben. Leseforum 2, 1-21.

Gladitz, Anne u.a. (2015): Alles unter DaF und Fach? Bestandsaufnahme, Handlungsbedarf und Vermittlungsansätze für Fachsprachenunterricht im internationalen Hochschulkontext. Ferraresi, Gisella/ Liebner, Sarah L. (Hg.): SprachBrückenBauen. Beiträge der 40. Jahrestagung DaF und DaZ an der Otto-Friedrich-Universität Bamberg. Göttingen: Bamberg Universitätsverlag, 149170.

Göpferich, Susanne (2016): Sich fachliches erschreiben: Förderung literaler Kompetenzen als Förderung des Denkens im Fach. Ballweg, Sandra (Hg.): Schreibberatung und Schreibförderung: Impulse aus Theorie, Empirie und Praxis.Frankfurt am Main: Peter Lang, 275-296.

Hatipoğlu, Sevinç (2015): Vergleich der Ergebnisse eines Projekts mit den Selbsteinschätzungen der angehenden türkischen Deutschlehrer in Bezug auf die Schreibkompetenz: Beispiel Istanbul Universität. Diyalog. Interkulturelle Zeitschrift für Germanistik 2, 44-56

Hayes, John R. (1996): A New Framework for Understanding Cognition and Affect in Writing. Levy, Micheal/ Ransdell, Sarah (Hg.): The Science of Writing. Theories, Methods, Individual Differences, and Applications. Mahwah: Lawrence Erlbaum Associates, 1-27.

Heinemann, Margot (2000): Textsorten des Bereichs Hochschule und Wissenschaft. Brinker, Klaus, u.a. (Hg.): Text- und Gesprächslinguistik. Ein internationales Handbuch zeitgenössischer Forschung.New York: De Gruyter, 702-710.

Janich, Nina (2008): Textlinguistik: 15 Einführungen. Tübingen: Gunter Narr Verlag.

Krumm, Hans-Jürgen (2014): Braucht es ,gute Lehrpersonen” für einen erfolgreichen Deutschunterricht?. AkDaF Rundbrief 67, 6-16. 
Kruse, Otto/ Ruhmann, Gabrielle (2006): Prozessorientierte Schreibdidaktik - Eine Einführung. Kruse, Otto/ Berger, Katja/ Ulmi, Marianne (Hg.): Prozessorientierte Schreibdidaktik: Schreibtraining für Schule, Studium und Beruf. Bern: Haupt Verlag, 13-38.

Kruse, Otto/ Perrin, Daniel (2002): Intuition und professionelles Schreiben. Das Thema dieses Buches. Perrin, Daniel, u.a. (Hg.): Schreiben - Von intuitiven zu professionellen Schreibstrategien. Wiesbaden: VS Verlag für Sozialwissenschaften, 7-13.

Leisen, Josef (2013): Handbuch Sprachförderung im Fach - Sprachensensibler Fachunterricht in der Praxis - Teilband: Praxismaterialien. Stuttgart: Ernst Klett Sprachen.

Lipowsky, Frank (2006): Auf den Lehrer kommt es an. Empirische Evidenzen für Zusammenhänge zwischen Lehrerkompetenzen, Lehrerhandeln und dem Lernen der Schüler. Allemann-Ghionda, Christina/ Terhart, Ewald (Hg.): Kompetenzen und Kompetenzentwicklung von Lehrerinnen und Lehrern. Weinheim: Beltz, 47-70.

Ludwig, Otto (1983): Einige Gedanken zu einer Theorie des Schreibens. Grosse, Siegfried (Hg.): Schriftsprachlichkeit. Düsseldorf: Pädagogischer Verlag Schwan, 37-73.

Lupo, Sarah M. u.a. (2012): Building Background Knowledge Through Reading: Rethinking Text Sets. Journal of Adolescent \& Adult Literacy61(4), 433-444.

Merz-Grötsch, Jasmin (2014): Texte schreiben lernen: Grundlagen, Methoden, Unterrichtsvorschläge. Bad Langensalza: Klett-Kallmeyer.

Ohm, Udo (2014): Ohne sprachliche Qualifizierung keine berufliche Qualifizierung. Zum konstitutiven Verhältnis zwischen der Aneignung von Fachwissen bzw. beruflicher Handlungskompetenz und Sprachentwicklung. Deutsch als Zweitsprache 1, 7-19.

Portmann-Tselikas, Paul R. (2005): Was ist Textkompetenz?. http://www.iagcovi.edu.gt/Homepagiag/paed/koord/deutsch/daf_2010/bewertung_dfu/textkompete nz_aufbau/PortmannTextkompetenz.pdf (Letzter Zugriff: 05.09.2020).

Portmann-Tselikas, Paul R./Schmözler-Eibinger, Sabine (2008): Textkompetenz. Fremdsprache Deutsch 39, 5-16.

Schmölzer-Eibinger, Sabine (2007): Auf dem Weg zur Literalen Didaktik. Schmözler-Eibinger, Sabine/ Weidacher, Georg (Hg.): Textkompetenz - Eine Schlüsselkompetenz und ihre Vermittlung. Tübingen: Gunter Narr Verlag, 207-222.

Schmölzer-Eibinger, Sabine (2008): Ein 3-Phasen-Modell zur Förderung der Textkompetenz. Fremdsprache Deutsch 39, 28-33.

Schmölzer-Eibinger, Sabine (2013): Sprache als Medium des Lernens im Fach. Becker-Mrotzek, Micheal, u.a. (Hg.): Sprache im Fach. Sprachlichkeit und fachliches Lernen. Münster: Waxmann, 25-40.

Seferoğlu, Sadi S. (2004): Öğretmen Yeterlilikleri ve Mesleki Gelişim. Bilim ve Aklın Aydınlı̆̆ında Ĕgitim 58, 40-45.

Sieber, Peter (2005): Didaktik des Schreibens - Vom Produkt zum Prozess und weiter zu Textkompetenz. Revue suisse des sciences de l'education27(3), 381-406.

Spivey, Nancy N. (1990): Transforming Texts. Written Communication 7(2), 256-287.

Stadter, Andrea (2003): Der Essay als Ziel und Instrument geisteswissenschaftlicher Schreibdidaktik. Überlegungen zur Erweiterung des universitären Textsortenkanons. Ehlich, Konrad/ Steets, Angelika (Hg.): Wissenschaftlich schreiben - lehren und lernen. Berlin: Walter de Gruyter, 65-92.

Tapan, Nilüfer (2000): Entwicklungen und Perspektiven der Deutschlehrerausbildung in der Türkei. Tapan, Nilüfer/ Polat, Tülin/ Schmidt, Hans-Werner (Hg.):Berufsbezogene Deutschlehrerausbildung. Istanbul: Türkischer Deutschlehrerverein, 37-44. 
Thonhauser, Ingo (2008): Textkompetenz im Fremdsprachenunterricht. Fremdsprache Deutsch 39, 1722.

Thonhauser, Ingo (2010): Textarbeit. Krumm, Hans-Jürgen, u.a. (Hg.): Deutsch als Fremd- und Zweitsprache. Ein internationales Handbuch. 1. Halbband. Hg. von.. Berlin, New York: De Gruyter Mouton, 1032-1037.

Vollmer, Helmut J.,/ Thürmann, Eike (2010): Zur Sprachlichkeit des Fachlernens: Modellierung eines Referenzrahmens für Deutsch als Zweitsprache. Ahrenholz, Bernt (Hg.): Fachunterricht und Deutsch als Zweitsprach. Tübingen: Narr Verlag, 107-132.

Witschel, Elfriede (2017): Textkompetenz fördern durch LesenSchreibenLesen - Die didaktische Bedeutung von Aufgabenarrangement im kompetenzorientierten Deutschunterricht. Frankfurt am Main: Peter Lang.

YÖK (2018): Yeni Öğretmen Yetiştirme Lisans Programları. https://www.yok.gov.tr/kurumsal/idaribirimler/egitim-ogretim-dairesi/yeni-ogretmen-yetistirme-lisans-programlari (letzter Zugriff: 13.07.2020). 